\title{
Ergodic Properties of a Semi-infinite Hard Rods System
}

\author{
O. DE PAZZIS \\ Observatoire de Meudon, Meudon, France
}

Received March 2, 1971

\begin{abstract}
A semi-infinite hard rods system in thermodynamic equilibrium is proved to be a $K$-system.
\end{abstract}

\section{§ 1. Introduction}

The ergodic hypothesis asserts that the asymptotic time average of any summable function is identical to its integral with respect to the probability measure governing the statistics of the system. It is easy to see that this last condition is equivalent to that all invariants under the evolution operator are constant "almost everywhere".

For finite classical systems the number of particles and the total energy are invariants under the evolution operator so that the canonical and grand-canonical equilibriums cannot be ergodic states. However the microcanonical equilibrium is expected to be ergodic for a large class of interaction potentials. In fact the ergodicity of the "Boltzmann-Gibbs" model (hard spheres model) has been recently proved by Sinaï [1].

Unfortunately the ergodicity of systems with a finite number of degrees of freedom is not of great interest in classical statistical mechanics. Indeed the thermodynamic limit gives rise to configurations with an infinite number of particles. In compensation one may conjecture that the thermodynamic equilibrium of infinite classical systems is ergodic (at least for a large class of potentials) for the following reason: the particle number and the total energy are not defined, so that one may think that constants are the only invariants. Precisely we propose in this paper to prove the ergodicity of a somewhat simple model in the thermodynamic limit.

A simple manner to describe a state of a system in the thermodynamic limit is to consider a probability measure on a space of so called "locally finite" configurations [2]. In this context the equilibrium state is unambiguously defined at low activity $z$ for a large class of interaction potentials [3]. Moreover in the case of hard core one-dimensional 
systems with reasonable interactions [4], the restriction on the activity can be omitted.

On the other hand the dynamical theory of configurations with infinite many particles has begun only recently. The first papers about this subject $[5,6]$ dealt only with one-dimensional systems with smooth and finite range potentials. In [5] the existence of the evolution operator $T^{t}$ was proved, on a subset $[\hat{X}]$ of the set of locally finite configurations $[X]$. In [6] the kinetic theory was developed. In particular the equilibrium state (at low activity) was shown to be concentrated on $[\hat{X}]$ and invariant under $T^{t}$.

Hence we have all the ingredients for tackling the question of the ergodicity of infinite one-dimensional systems. In fact the ergodicity of the infinite perfect gas has been recently proved by Sinaï et al. [7]. But to our knowledge the case of an infinite system with interactions has never been approached. The aim of this paper is precisely to treat the case of a very crude one-dimensional model which we describe now.

We consider a semi-infinite hard rods system on the positive semiaxis, with a perfectly reflecting wall at $q=-a / 2$, where $a$ is the common length of the rods. The collisions between the rods will be the only interactions considered. In $\S 2$ we describe the model and show that our dynamical system is isomorphic to the semi-infinite perfect gas with a perfectly reflecting wall at the origin. In $\S 3$ we recall the definition and the properties of $K$-systems which are basic systems in ergodic theory. Indeed $K$-systems are "mixing" and ergodic [8]. In $\S 4$ we follow very closely the arguments used in [7] and prove that the semi-infinite one-dimensional perfect gas in equilibrium is a $K$-system. The isomorphism mentioned above will ensure that our semi-infinite system of hard rods in equilibrium is also a $K$-system.

\section{$\S$ 2. Description of the Model}

Let $X_{a}$ be the space of sequences $x_{a}=\left\{q_{i}, p_{i}\right\}$ with

$$
q_{i} \geqq 0, p_{i} \in \mathscr{R} \quad \text { and } \quad\left|q_{i}-q_{j}\right| \geqq a \quad \text { if } i \neq j .
$$

Such sequences are infinite configurations of labelled hard rods. We say that two configurations are equivalent if they differ only by a permutation of the indexing set. We denote by $\left[X_{a}\right]$ the set of equivalence classes so that $\left[X_{a}\right]$ is the space of infinite configurations of unlabelled hard rods of length $a$. There is on $\left[X_{a}\right]$ a natural topology which makes $\left[X_{a}\right]$ a Polish space [6]. In the following no interactions other than collisions between the rods will be considered.

Let $K$ be the space obtained from $\left[X_{a}\right]$ by considering only the positions of the hard rods. $K$ is a compact space. $C(K)$ being the space of 
continuous functions on $K$, we consider the following family of operators defined on $C(K)$ for every values of $y \geqq 0$ :

$$
\left(\mathscr{L}_{y} f\right)(Y)=\int_{\substack{X \in K \\ X \cup X \subset\left[\tau_{y} Y \in, y \in\right.}} z^{N(X)} \cdot f\left(X \cup \tau_{y} Y\right) \cdot d X
$$

where $N(X)$ is the particle number of $X, \tau_{y}$ is the translation operator of modulus $y$ and $\int d X=\sum_{n} \int \frac{d q_{1} \cdot d q_{2} \ldots d q_{n}}{n !}$.

Let $P(z, \beta)$ be the thermodynamic pressure of the system at activity $z$ and temperature $\beta$. It is shown in [4] that the adjoint eigenvalue problem:

$$
\mathscr{L}_{y}^{*} \varrho=\exp (y \beta P(z, \beta)) \varrho
$$

has a unique solution which we denote by $\varrho_{z, \beta}^{a}$. In addition it is proved in [4] that $\varrho_{z, \beta}^{a}$ is obtained from the configurational grand-canonical ensemble by taking the thermodynamic limit.

According to the preceding results we shall call "thermodynamic equilibrium" of our model, at activity $z$ and temperature $\beta$, the state $\gamma_{z, \beta}^{a}$ on $\left[X_{a}\right]$ which has Maxwellian velocity distribution function at temperature $\beta$ and whose projection on $C(K)^{*}$ is given by $\varrho_{z, \beta}^{a}$.

The aim of this section is to prove that there exists an isomorphism between our hard rods model and the semi-infinite perfect gas which we describe now.

Let $[X]$ be the space of locally finite configurations in $\mathscr{R}^{+}$without hard core (as described in [6]). We call $\gamma_{z, \beta}$ the state on $[X]$ which has Maxwellian velocity distribution function at temperature $\beta$ and such that the probability density of having the configuration $q_{1}, q_{2}, \ldots q_{n}$ inside $[0, y)$ is given by: $\exp (-z y) \cdot z^{n} \cdot \frac{d q_{1} d q_{2} \ldots d q_{n}}{n !}$ for any $y \geqq 0$. It is clear that $\gamma_{z, \beta}$ is the thermodynamic equilibrium state at activity $z$ and temperature $\beta$ of the semi-infinite perfect gas.

We now show that there exists an isomorphism $\Pi$ between the two measured spaces: $\left(\left[X_{a}\right], \gamma_{z, \beta}^{a}\right)$ and $\left([X], \gamma_{z^{\prime}, \beta}\right)$ where $z^{\prime}$ is a given function of $z$.

First we construct a mapping from $\left[X_{a}\right]$ into $[X]$. Let $x_{a}=\left\{q_{i}, p_{i}\right\}$ be an element of $\left[X_{a}\right]$ after ordering the particles according to the increasing abscisses. We set:

$$
\Pi\left(x_{a}\right)=\left\{q_{i}^{\prime}, p_{i}^{\prime}\right\} \quad \text { with }\left\{\begin{array}{l}
q_{i}^{\prime}=q_{i}-(i-1) a \\
p_{i}^{\prime}=p_{i} .
\end{array}\right.
$$

This is a generalisation to the case of infinite configurations of a transformation already used in [9]. Of course $\Pi\left(x_{a}\right)$ does not belong to [X] for every $x_{a} \in\left[X_{a}\right]$; for instance consider $x_{a}=\left\{q_{i}, p_{i}\right\}$ with $q_{i}=i \cdot a$, then 
$\Pi\left(x_{a}\right)=\left\{q_{i}^{\prime}, p_{i}^{\prime}\right\}$ with $q_{i}^{\prime}=a$ for every $i$ and consequently $\Pi\left(x_{a}\right)$ is not a locally finite configuration. Moreover it is easy to see that $\Pi$ is not injective. Precisely we want to prove the three following points:

a) There exists Borel subsets $\left[\hat{X}_{a}\right]$ of $\left[X_{a}\right]$ and $[\hat{X}]$ of $[X]$ such that $\Pi$ is a Borel isomorphism from $\left[\hat{X}_{a}\right]$ into $[\hat{X}]$.

b) $\left[\hat{X}_{a}\right]$ is a set of measure one with respect to $\gamma_{z, \beta}^{a}$ for every values of $z$ and $\beta$.

c) $\gamma_{z, \beta}^{a} \circ \Pi^{-1}=\gamma_{z^{\prime}, \beta}$ where $z^{\prime}$ is a given function of $z$.

To this purpose we introduce the following notations:

$$
\left[\hat{X}_{a}\right]=\left(\left[X_{a}\right] \backslash\left[X_{a}^{c}\right]\right) \cap\left[\hat{X}_{a}^{1}\right]
$$

with:

$$
\begin{aligned}
& {\left[X_{a}^{c}\right]=\left\{\begin{array}{rr}
\text { either } \exists i, j:\left|q_{i}-q_{j}\right|=a \\
x_{a}=\left\{q_{i}, p_{i}\right\} ; & \text { and }\left(q_{i}-q_{j}\right)\left(p_{i}-p_{j}\right)<0 \\
& \text { or } \exists i: q_{i}=0 \text { and } p_{i}<0
\end{array}\right\}} \\
& {\left[\hat{X}_{a}^{1}\right]=\left\{x_{a} ; \sup _{i} \frac{\left|p_{i}\right|}{\log _{+}\left(q_{i}\right)} \vee \sup _{\alpha} \frac{\alpha}{\Gamma_{\alpha}(x)}<+\infty\right\}}
\end{aligned}
$$

where $\log _{+}(q)=\log (|q| \vee e), \Gamma_{\alpha}(x)=a \vee\left(\alpha-N_{[0, \alpha)}(x) \cdot a\right), N_{[0, \alpha)}(x)=$ the number of hard rods lying in $[0, \alpha)$ and $V$ means the supremum.

Let us comment on the above notations. We remark that $\left[X_{a}^{c}\right]$ is the set of configurations for which either two particles enter a collision with each other, or one particle enters a collision with the reflecting wall. We subtract $\left[X_{a}^{c}\right]$ from $\left[X_{a}\right]$ to make $\Pi$ injective. On the other hand $\left[\hat{X}_{a}^{1}\right]$ is the natural domain of definition of $\Pi$ and of the evolution operator $T^{t}$ as we will see later. The condition $\sup _{i} \frac{p_{i}}{\log ^{+}\left(q_{i}\right)}<+\infty$ has been already used in [5] and requires no further comments. On the other hand the condition $\sup _{\alpha} \frac{\alpha}{\Gamma_{a}(x)}<+\infty$ means that the residual volume between the rods in $[0, \alpha)$ is of the same order as $\alpha$ for large $\alpha$, or equivalently that the configuration $x$ does not have the "close-packing" density. This condition prevents catastrophic accumulations of rods during the evolution.

For analogous reasons we set [5]:

$$
[\hat{X}]=\left([X] \backslash\left[X^{c}\right]\right) \cap\left[\hat{X}^{1}\right]
$$

with $\left[X^{c}\right]=\left\{x \in[X] ; \exists i: q_{i}=0\right.$ and $\left.p_{i}<0\right\}$

$$
\left[\hat{X}^{1}\right]=\left\{x \in[X] ; \sup _{i} \frac{\left|p_{i}\right|}{\log _{+}\left(q_{i}\right)} \vee \sup _{\alpha} \frac{N_{[0, \alpha)}(x)}{\alpha}<+\infty\right\} .
$$

We now prove the main result of this section. 
Proposition 2.1. We have the following isomorphism (mod.0):

$$
\left(\left[X_{a}\right], \gamma_{z, \beta}^{a}\right) \stackrel{\Pi}{\longrightarrow}\left([X], \gamma_{z^{\prime}, \beta}\right)
$$

with $z^{\prime}=z \cdot \exp (-a \beta P(z, \beta))$.

Proof. a) By standard techniques one easily checks that $\left[\hat{X}_{a}\right]$ and $[\hat{X}]$ are Borel subsets of $\left[X_{a}\right]$ and $[X]$ respectively and that $\Pi$ is a Borel isomorphism from $\left[\hat{X}_{a}\right]$ onto $[\hat{X}]$.

b) We show that $\left[X_{a}\right] \backslash\left[\hat{X}_{a}\right]$ is a set of measure zero with respect to $\gamma_{z, \beta}^{a}$. The assertion that $\left[X_{a}^{c}\right]$ is a null set with respect to $\gamma_{z, \beta}^{a}$ follows directly from the fact that $\gamma_{z, \beta}^{a}$ has "local probability measures" absolutely continuous with respect to the Lebesgue measure. It is already proved in [6] that the set of configurations such that $\sup _{i} \frac{\left|p_{i}\right|}{\log _{+}\left(q_{i}\right)}=+\infty$ is a null set. Hence we have only to prove that the set of configurations such that $\sup _{n} \frac{n a}{\Gamma_{n a}(x)}=+\infty$ is a null set with respect to $\gamma_{z, \beta}^{a}$. In order to do this, we set:

$$
Y_{p}=\left\{x \in\left[X_{a}\right] ; \exists n>0: \frac{n a}{\Gamma_{n a}(x)} \geqq p\right\}
$$

It suffices to prove that $\lim _{p \rightarrow \infty} \gamma_{z, \beta}^{a}\left(Y_{p}\right)=0$. Let us introduce:

$$
Y_{p, N}=\left\{x \in\left[X_{a}\right] ; \frac{N a}{\Gamma_{N a}(x)} \geqq p\right\} .
$$

An elementary calculation leads to the following estimations:

$$
\gamma_{z, \beta}^{a}\left(Y_{p}\right) \leqq \sum_{N=1}^{\infty} \gamma_{z, \beta}^{a}\left(Y_{p, N}\right) \leqq \sum_{N=1}^{\infty} \sum_{n \geqq \frac{p-1}{p} \cdot N}^{N} \varrho_{N, n}
$$

where $\varrho_{N, n}$ is the probability of having $n$ particles in the interval $[0, N a$ ). We shall have an estimation of $\varrho_{N, n}$ in the following way. Let $\varrho_{N, n}(x)$ be the characteristic function of the set of configurations having $n$ particles in $[0, N a)$. We have:

$$
\varrho_{N, n}=\int \varrho_{N, n}(x) \cdot d \gamma_{z, \beta}^{a}=\exp (-N a \beta P(z, \beta)) \int \mathscr{L}_{N a}\left(\varrho_{N, n}\right)(x) \cdot d \gamma_{z, \beta}^{a} .
$$

But we have the following inequalities:

$$
0 \leqq \mathscr{L}_{N a} \varrho_{N, n}(x) \leqq z^{n} \cdot Q(N, n, \beta)
$$

where $Q(N, n, \beta)$ is the canonical partition function of $n$ hard rods in the interval $[0, N a$ ). In the absence of additional potential we have for 
$Q(N, n, \beta)$ the equation:

$$
Q(N, n, \beta)=\frac{((N+1-n) a)^{n}}{n !} .
$$

If we set $u_{N, n}=z^{n} \cdot Q(N, n, \beta)$, it results from (2.2) that there exists $p_{0}(z)$ such that if $p \geqq p_{0}(z), u_{N, n+1} \leqq u_{N, n}$ as soon as $n \geqq \frac{p-1}{p} \cdot N$. Hence for $p$ sufficiently large we get:

$$
\sum_{n \geqq \frac{p-1}{p} N}^{N} \varrho_{N, n} \leqq \frac{N}{p} \exp (-N a \beta P(z, \beta)) \cdot z^{\frac{p-1}{p} N} \cdot Q\left(N, \frac{p-1}{p} N, \beta\right) .
$$

Then using the definition of the free energy:

$$
f(\mu, \beta)=\lim _{\substack{N \rightarrow+\infty \\ n \rightarrow+\infty \\ \frac{n}{N a} \rightarrow \mu}}-\frac{\log (Q(N, n, \beta))}{N a \beta}
$$

we are led to the following estimation:

$$
\begin{aligned}
\gamma_{z, \beta}^{a}\left(Y_{p}\right) \leqq & \frac{\text { const. }}{p} \\
& \cdot \sum_{N=1}^{\infty} N \cdot \exp \left[\left(-\beta P(z, \beta)+\frac{p-1}{p} \log (z)-f\left(\frac{p-1}{p a}, \beta\right)\right) a N\right]
\end{aligned}
$$

if $p \geqq p_{0}(z)$. If we use the following definition of the thermodynamic pressure [10]:

$$
P(z, \beta)=\sup _{0 \leqq \mu \leqq \mu_{\text {c.p. }}}\left(\beta^{-1} \mu \log (z)-f(\mu, \beta)\right)
$$

and the convexity of $f(\mu, \beta)$ we finally have:

$$
\gamma_{z, \beta}^{a}\left(Y_{p}\right) \leqq \frac{\text { const. }}{p}
$$

for $p$ sufficiently large. Hence the assertion is proved ${ }^{1}$.

c) We now show that $\gamma_{z, \beta}^{a} \circ \Pi^{-1}=\gamma_{z^{\prime}, \beta}$ with $z^{\prime}=z \cdot \exp (-a \beta P(z, \beta))$. First we remark that $\Pi$ does not change the velocities so that $\gamma_{z, \beta}^{a} \circ \Pi$ has a Maxwellian velocity distribution function at temperature $\beta$. It suffices then to prove that the probability density $\tilde{\varrho}_{\alpha, n}\left(q_{1}, q_{2}, \ldots q_{n}\right)$ of having the configuration $\left(q_{1}, q_{2}, \ldots q_{n}\right)$ inside $[0, \alpha)$ with respect to $\gamma_{z, \beta}^{a} \circ \Pi^{-1}$ is

${ }^{1}$ A simpler argument based on a direct use of (2.2) can be given. Nevertheless we prefer the above argument because its generalisation to the case of an additional potential of the same type as in [4] is straightforward. 
given by:

$$
\tilde{\varrho}_{\alpha, n}\left(q_{1}, q_{2}, \ldots q_{n}\right)=\exp \left(-z^{\prime} \alpha\right) \cdot \frac{z^{\prime n} d q_{1} d q_{2} \ldots d q_{n}}{n !}
$$

with $z^{\prime}=z \cdot \exp (-a \beta P(z, \beta))$. By the very definition of $\Pi$ the probability density of having the configuration $\left(q_{1}, q_{2}, \ldots q_{n}\right)$ inside $[0, \alpha)$ with respect to $\gamma_{z, \beta}^{a} \circ \Pi^{-1}$ is exactly the probability density of having the configuration $\left(q_{1}, q_{2}+a, \ldots q_{n}+(n-1) a\right)$ inside $[0, \alpha+n a)$ with respect to $\gamma_{z, \beta}^{a}$. Then using equation (2.1) with $y=\alpha+n a$, we get:

$$
\tilde{\varrho}_{\alpha, n}\left(q_{1}, q_{2} \ldots q_{n}\right)=\exp (-\alpha \beta P(z, \beta)) \cdot \frac{\left(\alpha z \cdot \exp (-a \beta P(z, \beta))^{n}\right.}{n !} d q_{1} \ldots d q_{n} .
$$

This completes the proof of the proposition. However we notice that we obtained an additional result. In fact the normalisation condition $\sum_{n} \int \tilde{\varrho}_{\alpha, n}\left(q_{1}, q_{2}, \ldots q_{n}\right) d q_{1} d q_{2} \ldots d q_{n}=1$ implies the following implicit equation defining the thermodynamic pressure:

$$
\beta P(z, \beta)=z \cdot \exp (-a \beta P(z, \beta)) .
$$

To conclude this section we have still to define the evolution operator $T_{a}^{t}$ on $\left[X_{a}\right]$. In order to do this we remark that a collision between two hard rods merely interchanges their velocities, so that if we know the evolution operator $T^{t}$ of the semi-infinite perfect gas with a reflecting wall at the origin, we shall have $T_{a}^{t}$ by the equation:

$$
T_{a}^{t}=\Pi^{-1} \circ T^{t} \circ \Pi \text {. }
$$

But the problem of the existence of $T^{t}$ on $[\hat{X}]$ is a very simple one. Although the results of [5] cannot be directly applied because a slightly more restrictive condition than $\sup _{\alpha} \frac{N_{[0, \alpha)}(x)}{\alpha}<+\infty$ is used in [5] for proving the existence of $T^{t}$, it is easy to prove the following lemma:

Lemma 2.2. $T^{t}$ is a one-parameter group of Borel isomorphisms from $[\hat{X}]$ onto $[\hat{X}], \gamma_{z, \beta}$ is concentrated on $[\hat{X}]$ and is invariant under $T^{t}$.

Hence we can resume all the results of this section in the following proposition:

Proposition 2.3. $\Pi$ is an isomorphism of dynamical systems:

$$
\left(\left[X_{a}\right], \gamma_{z, \beta}^{a}, T_{a}^{t}\right) \stackrel{\Pi I}{\longleftrightarrow}\left([X], \gamma_{z^{\prime}, \beta}, T^{t}\right) .
$$

with $z^{\prime}=z \cdot \exp (-a \beta P(z, \beta))$. 


\section{§ 3. The $K$-systems}

For the terminology and the fundamental concepts used in the following, the reader is referred to the book of Arnold and Avez [8] and the basic paper of Rohlin [11]. In this section, we give only the fundamental definitions which are essential to understand the rest of this paper. A dynamical system will be represented by a triplet $\left(X, \gamma, T^{t}\right)$ where $X$ is a space, $\gamma$ a probability measure on $X$ and $T^{t}$ is a one-parameter group of isomorphisms (mod $\cdot 0)$ of $X$ onto itself, leaving $\gamma$ invariant. We start with the definition of an ergodic dynamical system:

Definition 3.1. The dynamical system $\left(X, \gamma, T^{t}\right)$ is said to be ergodic if for every $f(x) \in L^{1}(X, \gamma)$ we have:

$$
\lim _{t \rightarrow+\infty} \frac{1}{t} \int_{0}^{t} f\left(T^{\tau} x\right) \cdot d \tau=\int f \cdot d \gamma
$$

for almost every $x$ with respect to $\gamma$.

As the aim of $\S 4$ is to prove that our dynamical system $\left(\left[X_{a}\right], \gamma_{z, \beta}^{a}, T_{a}^{t}\right)$ is a $K$-system, which is a much stronger property, we must give the definition of $K$-systems.

Definition 3.2. A dynamical system $\left(X, \gamma, T^{t}\right)$ is called a $K$-system if: $-(X, \gamma)$ is a continuous Lebesgue space, that is $(X, \gamma)$ is isomorphic $(\bmod \cdot 0)$ to the unit interval $[0,1]$ equipped with the Lebesgue measure.

- there exists a "measurable decomposition" $\mathscr{J}_{0}$ of $X$ such that:
a) $T^{t} \mathscr{J}_{0}=\mathscr{J}_{t} \geqq \mathscr{J}_{0} \quad(\bmod \cdot 0)$ if $t \geqq 0$,
b) $\bigvee \mathscr{J}_{t}=\varepsilon(\bmod \cdot 0)$
c) $\bigwedge_{t}^{t} \mathscr{J}_{t}=v \quad(\bmod \cdot 0)$

where $\varepsilon$ is the decomposition of $X$ whose elements are the points of $X$ $X$, and $v$ is the trivial decomposition of $X$ whose unique element is $X$ itself. We recall that (mod.0) means "up to a null set with respect to $\gamma^{\prime \prime}$.

The above definition requires some comments. In fact the condition a) implies that the decomposition $\mathscr{J}_{t}$ is finer than $\mathscr{J}_{0}$ if $t \geqq 0$, whereas the condition b) means that if two configurations are in the same component of $\mathscr{J}_{0}$ at time $t=0$, then their "mutual distance" vanishes as $t \rightarrow+\infty$. Condition c) means that any measurable set invariant under $T^{t}$ is either a null set or a set of measure one; in other words, condition c) is the ergodic property.

Finally we recall the following sequence of implications:

$$
K \text {-system } \Rightarrow\left\{\begin{array}{l}
\text { system with countably } \\
\text { multiple Lebesgue spectra }
\end{array}\right\} \Rightarrow \text { mixing } \Rightarrow \text { ergodic . }
$$




\section{§ 4. Ergodic Properties of $\left(\left[X_{a}\right], \gamma_{z, \beta}^{a}, T_{a}^{t}\right)$}

In order to prove that $\left(\left[X_{a}\right], \gamma_{z, \beta}^{a}, T_{a}^{t}\right)$ is a $K$-system, we begin by proving that $\left([X], \gamma_{z, \beta}, T^{t}\right)$ is a $K$-system, then the use of the isomorphism $\Pi$ will lead to the desired result. Let us state the main result of this section, whose proof is very close to that used in [7] for proving that the infinite perfect gas in equilibrium is a $K$-system:

Proposition 4.1. The dynamical system $\left([X], \gamma_{z, \beta}, T^{t}\right)$ is a $K$-system.

Proof. First we have to verify that $\left([X], \gamma_{z, \beta}\right)$ is a continuous Lebesgue space. This results from the fact that $[X]$ is a Polish space and $\gamma_{z, \beta}$ a continuous Borel probability measure on $[X]$ and from the following lemma (proved in [11], p. 24): Lemma: Every measured space $(X, \gamma)$ where $X$ is a Polish space and $\gamma$ a continuous Borel probability measure on $X$ is a continuous Lebesgue space.

We describe now the decomposition $\mathscr{J}_{0}$. Let $x$ be an element of $[X]$. We denote by $\tilde{x}$ the configuration obtained from $x$ by keeping only the particles with negative velocities. We say that $x_{1}$ and $x_{2}$ are in the same component of $\mathscr{J}_{0}$ if and only if $\tilde{x}_{1}=\tilde{x}_{2}$.

First we have to show that $\mathscr{J}_{0}$ is a "measurable decomposition". We denote by $\left\{C_{i}\right\}, i \in I$, the components of $\mathscr{J}_{0}$. We must prove that there exists a countable family $\left\{B_{n}\right\}$ of measurable sets such that every $B_{n}$ is a union of components $C_{i}$ and that for every pair $C_{i}, C_{j}$ with $i \neq j$, there exists a $B_{n}$ for which:

$$
\begin{array}{rll}
\text { either } C_{i} \sqsubset B_{n} & \text { and } & C_{j} \not \subset B_{n} \\
\text { or } C_{j} \sqsubset B_{n} & \text { and } & C_{i} \not \subset B_{n} .
\end{array}
$$

Let us call $J$ the set of all pairs $\{[\alpha, \beta],[\gamma, \delta]\}$ where $\alpha, \beta \geqq 0 \gamma, \delta \leqq 0$ and $\alpha, \beta, \gamma, \delta$ are rational numbers. $J$ is a countable set. Let $j=\{[\alpha, \beta],[\gamma, \delta]\}$ an element of $J$, we call $B_{j}$ the set of configurations such that there is at least one particle lying in $[\alpha, \beta]$ and having a velocity lying in $[\gamma, \delta]$. It is easy to see that the family $\left\{B_{n}\right\}$ satisfies all the above conditions. This proves the assertion. We must now prove that $\mathscr{J}_{0}$ fulfils the conditions a), b) and c) of a $K$-system.

Condition a) is obvious. Condition b) will be verified if the set of configurations such that there is at least one particle with a nul velocity is a null set. This last property follows from the fact that $\gamma_{z, \beta}$ has a Maxwellian velocity distribution function. Then it remains to prove condition c). At this stage we follow very closely the arguments used in [7]. Consequently we will recall only the fundamental steps of the proof.

Let us choose a particular sequence $t_{n} \rightarrow+\infty$. We set:

$$
\mathscr{J}_{n}=\mathscr{J}_{-t_{n}} \text { and } \mathscr{J}=\bigwedge_{n} \mathscr{J}_{n} .
$$


It suffices to prove that:

$$
\mathscr{J}=v \quad(\bmod \cdot 0) .
$$

In order to do this we remark that (4.1) is equivalent to:

$$
\left\{\begin{array}{l}
\text { "for any measurable set } A ; \quad \gamma_{z, \beta}\left(A \mid C_{\mathscr{g}}(x)\right)=\gamma_{z, \beta}(A) \\
\text { for almost every } x \text { " }
\end{array}\right\}
$$

where $C_{\mathscr{f}}(x)$ denotes the component of $\mathscr{J}$ containing $x$ and $\gamma_{z, \beta}\left(A \mid C_{\mathscr{J}}(x)\right)$ is the "conditional measure" of $A$ with respect to the component $C_{\mathscr{f}}(x)$ [11].

We shall now use the "quasi-local" property of the $\sigma$-algebra of measurable sets. More precisely, let $\alpha$ be any positive number, we call $\mathscr{A}_{\alpha}$ the $\sigma$-algebra of the sets which are measurable inside $[0, \alpha$ ), (i.e. whose characteristic function is measurable inside $[0, \alpha)$ ). We have the identity:

$$
\bigvee_{\alpha} \mathscr{A}_{\alpha}=\mathscr{A}
$$

where $\mathscr{A}$ is the $\sigma$-algebra of all the measurable sets. The statement (4.2) is then equivalent to:

$$
\left\{\begin{array}{l}
\text { "for any locally measurable set } A ; \quad \gamma_{z, \beta}\left(A \mid C_{\mathscr{J}}(\mathrm{x})\right)=\gamma_{z, \beta}(A) \\
\text { for almost every } x "
\end{array}\right\} .
$$

At this step we recall the Doob's theorem which asserts that if $\mathscr{J}_{n} \downarrow$ and $\mathscr{J}=\bigwedge_{n} \mathscr{J}_{n}$ then for any $A \in \mathscr{A}$ :

$$
\lim _{n \rightarrow+\infty} \gamma_{z, \beta}\left(A \mid C_{\mathscr{g}_{n}}(x)\right)=\gamma_{z, \beta}\left(A \mid C_{\mathscr{g}}(x)\right) \text { for almost every } x .
$$

As a consequence, in order to prove (4.3) it suffices to show that:

$$
\left\{\begin{array}{l}
\text { "for any locally measurable set } A ; \\
\lim _{n \rightarrow+\infty} \gamma_{z, \beta}\left(A \mid C_{\mathscr{\not}_{n}}(x)\right)=\gamma_{z, \beta}(A)
\end{array}\right\}
$$

In the remaining $A$ will be an element of $\mathscr{A}_{\alpha}$ for a given $\alpha \geqq 0$.

Let us introduce the following set:

$$
\mathscr{N}_{n}(\alpha)=\left\{x \in[X] ;\left|p_{i}\right|>\frac{\alpha}{t_{n}} \quad \text { if } \quad q_{i} \in[0, \alpha)\right\} .
$$

Using the fact that the conditional measures $\gamma_{z, \beta}\left(\mid C_{\mathscr{f}}\right)$ have Maxwellian velocity distribution functions it is easy to see that:

$$
\lim _{n \rightarrow \infty} \gamma_{z, \beta}\left(A \cap \overline{\mathscr{N}_{n}(\alpha)} \mid C_{\mathscr{J}_{n}}(x)\right)=0 \quad \text { for almost every } x \in \mathscr{N}_{n}(\alpha) .
$$


Here $\bar{A}$ denotes the complement of $A$. Hence (4.4) reduces to:

$$
\lim _{n \rightarrow \infty} \gamma_{z, \beta}\left(A \cap \mathscr{N}_{n}(\alpha) \mid C_{\mathscr{g}_{n}}(x)\right)=\gamma_{z, \beta}(A) \quad \text { for almost every } x .
$$

We remark that if $x \in \mathscr{N}_{n}(\alpha)$ then $C_{\mathscr{g}_{n}}(x)$ implies some conditions outside $[0, \alpha)$ only. The absence of correlations between two disjoint intervals leads to:

$$
\gamma_{z, \beta}\left(A \cap \mathscr{N}_{n}(\alpha) C_{\mathscr{f}_{n}}(x)\right)=\gamma_{z, \beta}\left(A \cap \mathscr{N}_{n}(\alpha)\right) \quad \text { for almost every } \quad x \in \mathscr{N}_{n}(\alpha) .
$$

The proof of the proposition is completed if we remark that:

$$
\lim _{n \rightarrow+\infty} \gamma_{z, \beta}\left(A \cap \mathcal{N}_{n}(\alpha)\right)=\gamma_{z, \beta}(A) .
$$

Corollary. The dynamical system $\left(\left[X_{a}\right], \gamma_{z, \beta}^{a}, T_{a}^{t}\right)$ is a K-system.

Proof. Use the Proposition 2.3 and the fact that every dynamical system which is isomorphic to a $K$-system is also a $K$-system.

\section{§ 5. Remarks}

We give here some comments on the preceding results. First we mention that the above corollary implies that the diffusion coefficient for the system at hand is zero. In fact, if we label the particle nearest to the wall by 1 , it is easy to show that $q_{1}(x)$ and $p_{1}(x)$ are square integrable functions with respect to $\gamma_{z, \beta}^{a}$. Hence we can apply the mixing property, that is:

$$
\lim _{T \rightarrow+\infty} \int_{0}^{T}\left\langle p_{1}(t) \cdot p_{1}(0)\right\rangle d t=\lim _{T \rightarrow+\infty} m\left\langle q_{1}(T) \cdot p_{1}(0)\right\rangle=0
$$

and this shows that the diffusion coefficient is zero. This is not a surprising result because if the particle 1 could diffuse far from the wall, the vacuum could occupy all the volume, which is impossible if $z>0^{2}$.

We have succeded in proving that our hard rods system is ergodic by the use of an artifice, more precisely by showing that the system was isomorphic to the semi-infinite perfect gas. The existence of this isomorphism is directly related to the fact that our model is not "dissipative", that is the homogeneous Boltzmann collision operator reduces to zero. As a consequence, strictly speaking, there is no hydrodynamical description of the model and it is clear that this limits somewhat the interest of the model.

Nevertheless it would be interesting to prove that the infinite hard rods system is ergodic too. Unfortunately the existence of an isomorphism

${ }^{2}$ It is gratefully acknowledged that this remark on the diffusion coefficient follows from a discussion with Prof. J. Lebowitz. 
like $\Pi$ seems more difficult to prove, so that a direct proof of the ergodicity of the model must be investigated. The main difficulty which arises then is the existence of correlations, so that the conditional measures associated with a measurable decomposition $\mathscr{J}$ are not simple to describe. However it is established in the case of one-dimensional hard-core systems that the equilibrium state has the "cluster" property [4]. Using this remark it has been possible to prove that the infinite hard rods system with discrete velocities $p= \pm p_{0}$ is a $K$-system.

Note added in proof: Recently Professor Sinai informed the present author that he has solved the problem of the infinite system with maxwellian velocity distribution.

\section{References}

1. Sinaï, Ya.: Sov. Math. Dokl. 4, 1818 (1963).

2. Ruelle, D.: J. Math. Phys. 8, 1657 (1967).

3. - Ann. Phys. 25, 109 (1963).

4. Gallavotti, G., Miracle-Sole, S.: J. Math. Phys. 11, 147 (1969).

5. Lanford, III, O. E.: Commun. math. Phys. 9, 176 (1968).

6. - Commun. math. Phys. 11, 257 (1969).

7. Sinaï, Ya., Volkoviskij, K.L.: Ergodic properties of the perfect gas with infinite many degrees of freedom (unpublished).

8. Arnold,V.I., Avez,A.: Problemes ergodiques de la mécanique classique. Paris: Gauthiers-Villars 1967.

9. Lebowitz, J. L., Percus, J. K., Sykes, J.: Phys. Rev. 171, 224 (1968).

10. Ruelle,D.: Statistical mechanics. New York: Benjamin 1969.

11. Rohlin, V.A.: Amer. Math. Soc. Transl. (1) 10, 1-54 (1962).

O. de Pazzis

Observatoire de Meudon

Department of Physics

F-92 Meudon, France 